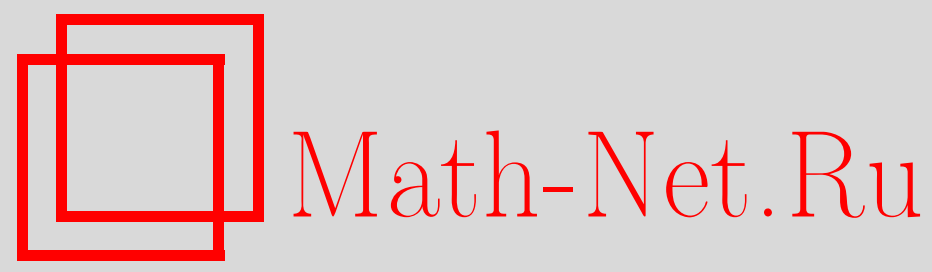

Р. М. Колпаков, М. А. Посыпкин, Верхняя и нижняя оценки трудоемкости метода ветвей и границ для задачи о ранце, Дискрет. матем., 2010, том 22, выпуск 1, 58-73

DOI: https://doi.org/10.4213/dm1084

Использование Общероссийского математического портала Math-Net.Ru подразумевает, что вы прочитали и согласны с пользовательским соглашением http://www . mathnet.ru/rus/agreement

Параметры загрузки:

IP: 35.174 .16 .151

26 апреля 2023 г., $11: 34: 35$ 


\title{
Верхняя и нижняя оценки трудоемкости метода ветвей и границ для задачи о ранце
}

\author{
(c) 2010 г. Р. М. Колпаков, М. А. Посыпкин
}

\begin{abstract}
Статья посвящена вопросам сложности решения задачи об одномерном булевом ранце методом ветвей и границ. Для рассматриваемой сложности получены две верхние оценки. Выделен частный случай задачи о ранце, когда сложность ее решения полиномиально ограничена размерностью задачи. Получены также верхняя и нижняя оценки сложности решения методом ветвей и границ задачи о сумме подмножеств, являющейся частным случаем задачи о ранце.

Работа выполнена при поддержке Российского фонда фундаментальных исследований, проекты 05-01-00495a, 06-07-89079а.
\end{abstract}

\section{1. Введение}

В данной работе рассматривается задача о ранце, которая формулируется следующим образом. Даны $n$ предметов, при этом $i$-й предмет характеризуется весом $w_{i}$ и ценой $p_{i}$. Требуется положить в ранец грузоподъемностью $C$ набор предметов максимальной стоимости. Задача о ранце является одной из классических задач дискретной оптимизации, исследованию которой посвящены многочисленные статьи и монографии $[2,3]$.

В настоящее время существуют различные алгоритмы решения задачи о ранце, среди которых одними из наиболее распространенных являются методы ветвей и границ (МВГ). Эти методы основаны на древовидной декомпозиции множества допустимых решений. За счет исключения из рассмотрения подзадач, решения которых заведомо не являются оптимальными, часто удается существенно снизить объем вычислений по сравнению с полным перебором.

Особенностью метода ветвей и границ является то, что трудоемкость решения задачи этим методом существенно зависит от входных данных задачи. В частности, в [8] приведен пример задачи о ранце, сложность решения которого, определяемая числом рассматриваемых в процессе решения подзадач, имеет порядок $2^{n} / \sqrt{n}$. В то же время существует тривиальный пример задачи о ранце произвольной размерности, для решения которой требуется один шаг МВГ. Поэтому важным является получение оценок сложности решения задачи, зависящих от входных данных задачи. Такие оценки позволяют сделать определенные выводы о трудоемкости задачи, не решая ее.

В настоящей работе для задачи о ранце получены две верхние оценки сложности ее решения методом ветвей и границ, зависящие от входных данных задачи. Выделен частный случай, когда сложность решения задачи о ранце методом ветвей и границ полиномиально ограничена размерностью задачи. Получены также верхняя и нижняя оценки 
сложности решения методом ветвей и границ задачи о сумме подмножеств, являющейся важным частным случаем задачи о ранце.

\section{2. Некоторые свойства би-деревьев}

В этом разделе устанавливаются некоторые свойства деревьев определенного вида, которые будут необходимы в дальнейшем для получения оценок трудоемкости метода ветвей и границ.

Определение 1. Би-деревом будем называть ориентированное бинарное дерево с корнем, обладающее следующими свойствами:

(1) каждая дуга помечена 0 или 1 ;

(2) из каждой внутренней (в том числе корневой) вершины исходит ровно две дуги, помеченные 0 и 1 соответственно;

(3) в каждую вершину, отличную от корневой, входит ровно одна дуга.

Определение 2. Пусть $a-$ вершина би-дерева $T$. Поддерево $S$ с корнем $a$ би-дерева $T$ будем называть би-поддеревом с корнем $a$ би-дерева $T$, если $S$ является би-деревом. Если $а$ при этом является корнем дерева $T$, то будем называть $S$ главным би-поддеревом би-дерева $T$.

Определение 3. Пусть $a$ - вершина би-дерева $T$. Би-поддерево $S$ с корнем $a$ би-дерева $T$ будем называть максимальным, если любое би-поддерево с корнем $a$ би-дерева $T$ является также би-поддеревом дерева $S$.

Определим следующие подмножества вершин би-дерева:

$V(T)$ - множество вершин би-дерева $T$;

$V_{*}(T)-$ множество концевых вершин би-дерева $T$;

$V_{*}^{0}(T)$ - множество концевых вершин би-дерева $T$, в которые входит дуга, помеченная 0 ;

$V_{*}^{1}(T)$ - множество концевых вершин би-дерева $T$, в которые входит дуга, помеченная 1.

Введем также следующие обозначения для мощностей перечисленных множеств:

$$
\begin{aligned}
v(T) & =|V(T)|, & & v_{*}(T)=\left|V_{*}(T)\right|, \\
v_{*}^{0}(T) & =\left|V_{*}^{0}(T)\right|, & & v_{*}^{1}(T)=\left|V_{*}^{1}(T)\right| .
\end{aligned}
$$

Если $a-$ некоторая вершина би-дерева $T$, то последовательность дуг, соединяющую корень дерева $T$ с этой вершиной, будем обозначать через $\pi(a)$. Число дуг в этой последовательности, помеченных 0 , обозначим через $e_{0}(a)$, а число дуг, помеченных $1,-$ через $e_{1}(a)$.

Определение 4. Будем называть $\left(c_{0}, c_{1}\right)$-деревом би-дерево $T$ такое, что

(1) $e_{0}(x)=c_{0}$ для всех вершин $x$, принадлежащих $V_{*}^{0}(T)$;

(2) $e_{1}(x)=c_{1}$ для всех вершин $x$, принадлежащих $V_{*}^{1}(T)$. 


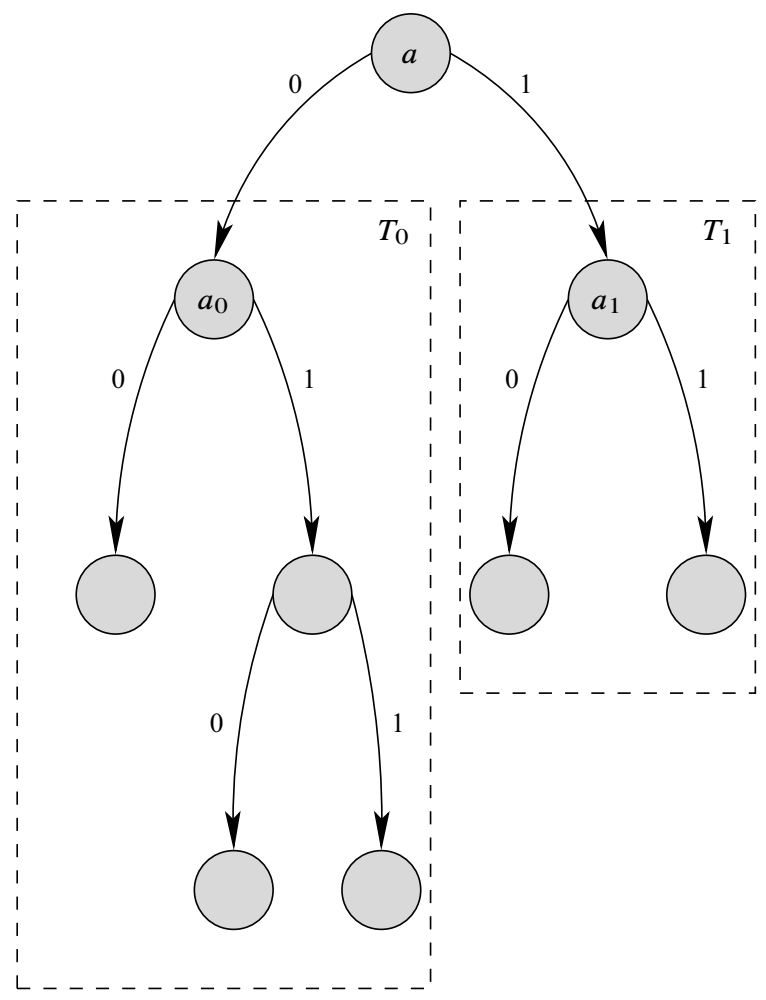

Рис. 1. Декомпозиция би-дерева $T$ на би-деревья $T_{0}$ и $T_{1}$

Заметим, что $\left(c_{0}, c_{1}\right)$-дерево можно построить для любых натуральных $c_{0}, c_{1}$.

Предложение 1. Пусть $T-\left(c_{0}, c_{1}\right)$-дерево. Тогда

$$
v_{*}(T)=\left(\begin{array}{c}
c_{0}+c_{1} \\
c_{0}
\end{array}\right)
$$

Доказательство. Доказательство проведем индукцией по величине $c_{0}+c_{1}$. В случае, когда $c_{0}=1$ или $c_{1}=1$, утверждение очевидно. Пусть для некоторого натурального $c>3$ утверждение справедливо для всех $\left(c_{0}, c_{1}\right)$-деревьев таких, что $c_{0}+c_{1}<c$. Рассмотрим произвольное $\left(c_{0}, c_{1}\right)$-дерево $T$ такое, что $c_{0}, c_{1}>1$ и $c_{0}+c_{1}=c$. Пусть $a_{0}$ и $a_{1}-$ вершины, соединенные с корнем $a$ дерева $T$ дугами, помеченными 0 и 1 соответственно (рис. 1).

Обозначим через $T_{0}$ и $T_{1}$ максимальные би-поддеревья би-дерева $T$ с корнями $a_{0}$ и $a_{1}$ соответственно. Нетрудно заметить, что дерево $T_{0}$ является $\left(c_{0}-1, c_{1}\right)$-деревом, а дерево $T_{1}$ является $\left(c_{0}, c_{1}-1\right)$-деревом. Поэтому, согласно индуктивному предположению,

$$
v_{*}\left(T_{0}\right)=\left(\begin{array}{c}
c_{0}+c_{1}-1 \\
c_{0}-1
\end{array}\right), \quad v_{*}\left(T_{1}\right)=\left(\begin{array}{c}
c_{0}+c_{1}-1 \\
c_{0}
\end{array}\right) .
$$


Следовательно,

$$
\begin{aligned}
v_{*}(T) & =v_{*}\left(T_{0}\right)+v_{*}\left(T_{1}\right) \\
& =\left(\begin{array}{c}
c_{0}+c_{1}-1 \\
c_{0}-1
\end{array}\right)+\left(\begin{array}{c}
c_{0}+c_{1}-1 \\
c_{0}
\end{array}\right)=\left(\begin{array}{c}
c_{0}+c_{1} \\
c_{0}
\end{array}\right) .
\end{aligned}
$$

Тем самым утверждение доказано.

Предложение 2. Пусть $T$ - би-дерево, для которого справедливы следующие соотношения:

(1) $e_{0}(x) \geqslant c_{0}$ для всех вершин $x$, принадлежащчих $V_{*}^{0}(T)$;

(2) $e_{1}(x) \geqslant c_{1}$ для всех вершин $x$, принадлежащчих $V_{*}^{1}(T)$.

Тогда

$$
v_{*}(T) \geqslant\left(\begin{array}{c}
c_{0}+c_{1} \\
c_{0}
\end{array}\right) .
$$

Доказательство. Выделим в $T$ главное $\left(c_{0}, c_{1}\right)$-поддерево $T^{*}$ следующим образом. Обозначим

$$
\begin{aligned}
& E_{0}(T)=\left\{x \in V_{*}^{0}(T) \mid e_{0}(x)>c_{0}\right\}, \\
& E_{1}(T)=\left\{x \in V_{*}^{1}(T) \mid e_{1}(x)>c_{1}\right\} .
\end{aligned}
$$

Если $E_{0}(T)=\varnothing$ и $E_{1}(T)=\varnothing$, то положим $T^{*}=T$. Пусть $E_{0}(T) \neq \varnothing$. Рассмотрим вершину $x \in E_{0}(T)$. Существует вершина $x^{\prime}, x^{\prime} \in \pi(x)$, такая, что $e_{0}(x)=c_{0}$ и дуга, входящая в $x^{\prime}$, помечена 0. Рассмотрим главное би-поддерево $T^{\prime}$ дерева $T$, получаемое удалением из $T$ максимального би-поддерева с корнем $x^{\prime}$. Дерево $T^{\prime}$ удовлетворяет соотношениям (1)-(2) доказываемого предложения, так как все его концевые вершины, кроме $x^{\prime}$, также являются концевыми вершинами дерева $T$, а для вершины $x^{\prime}$ справедливо равенство $e_{0}\left(x^{\prime}\right)=c_{0}$. Заметим, что

$$
\left|E_{0}\left(T^{\prime}\right)\right| \leqslant\left|E_{0}(T)\right|-1
$$

Поэтому, повторяя данную процедуру не более чем $\left|E_{0}(T)\right|$ раз, получим поддерево $T^{\prime \prime}$ бидерева $T$, удовлетворяющее соотношениям (1)-(2) доказываемого предложения и условию $\left|E_{0}\left(T^{\prime \prime}\right)\right|=0$. Аналогично построим поддерево $T^{\prime \prime \prime}$ би-дерева $T^{\prime \prime}$, удовлетворяющее соотношениям (1)-(2) доказываемого предложения и условиям $E_{0}\left(T^{\prime \prime \prime}\right)=\varnothing, E_{1}\left(T^{\prime \prime \prime}\right)=\varnothing$, и положим $T^{*}=T^{\prime \prime \prime}$. Согласно предложению 1 ,

$$
v_{*}\left(T^{*}\right)=\left(\begin{array}{c}
c_{0}+c_{1} \\
c_{0}
\end{array}\right) .
$$

Так как $T^{*}-$ поддерево дерева $T$, справедливо неравенство

$$
v_{*}(T) \geqslant v_{*}\left(T^{*}\right) .
$$

Следовательно,

$$
v_{*}(T) \geqslant\left(\begin{array}{c}
c_{0}+c_{1} \\
c_{0}
\end{array}\right) .
$$


Предложение 3. Пусть $T$ - би-дерево, для которого выполняются следующие условия:

(1) $e_{0}(x) \leqslant c_{0}$ для всех вершин $x$, принадлежащчих $V_{*}^{0}(T)$;

(2) $e_{1}(x) \leqslant c_{1}$ для всех вершин $x$, принадлежащчих $V_{*}^{1}(T)$.

Тогда

$$
v_{*}(T) \leqslant\left(\begin{array}{c}
c_{0}+c_{1} \\
c_{0}
\end{array}\right) .
$$

Доказательство. Для любого би-дерева $T$, удовлетворяющего условиям (1)-(2), обозначим

$$
\begin{aligned}
& E_{0}(T)=\left\{x \in V_{*}^{0}(T) \mid e_{0}(x)<c_{0}\right\}, \\
& E_{1}(T)=\left\{x \in V_{*}^{1}(T) \mid e_{1}(x)<c_{1}\right\} .
\end{aligned}
$$

Покажем, что дерево $T$ является главным поддеревом некоторого $\left(c_{0}, c_{1}\right)$-дерева $T^{*}$. Если $E_{0}(T)=\varnothing$ и $E_{1}(T)=\varnothing$, то $T^{*}=T$. Пусть $E_{0}(T) \neq \varnothing$. Рассмотрим вершину $x \in E_{0}(T)$. Пусть $y-$ вершина, из которой исходит дуга, ведущая в $x$. Очевидно, что $e_{1}(x)=e_{1}(y)$. Поскольку из $y$ исходит также дуга, помеченная 1 , справедливо неравенство $e_{1}(y)<c_{1}$. Поэтому $e_{1}(x)<c_{1}$. Справедливо также неравенство $e_{0}(x)<c_{0}$. Положим $d_{0}=c_{0}-e_{0}(x), d_{1}=c_{1}-e_{1}(x)$. Пусть $S$ - произвольное $\left(d_{0}, d_{1}\right)$-дерево. Построим бидерево $T^{\prime}$ добавлением к дереву $T$ дерева $S$, совместив $x$ с корнем дерева $S$. В результате концевая вершина $x$ в $T$ заменится $v_{*}(S)$ концевыми вершинами в $T^{\prime}$, и для каждой вершины $x^{\prime}$ из этих концевых вершин выполняется равенство $e_{0}\left(x^{\prime}\right)=c_{0}$, если $x^{\prime} \in E_{0}\left(T^{\prime}\right)$, и равенство $e_{1}\left(x^{\prime}\right)=c_{1}$, если $x^{\prime} \in E_{1}\left(T^{\prime}\right)$. Следовательно, $\left|E_{0}\left(T^{\prime}\right)\right|=\left|E_{0}(T)\right|-1$ и $\left|E_{1}\left(T^{\prime}\right)\right|=\left|E_{1}(T)\right|$. Повторяя данную процедуру $\left|E_{0}(T)\right|$ раз для каждой вершины из $E_{0}(T)$, получим би-дерево $T^{\prime \prime}$, содержащее $T$ в качестве главного поддерева и удовлетворяющее условиям (1)-(2) и соотношению $\left|E_{0}\left(T^{\prime \prime}\right)\right|=0$. Аналогичную процедуру можно также применить для каждой вершины из $E_{1}(T)$. В результате получим $\left(c_{0}, c_{1}\right)$-дерево $T^{*}$, содержащее дерево $T$ в качестве главного поддерева. Очевидно, что $v_{*}(T) \leqslant v_{*}\left(T^{*}\right)$. Согласно предложению 1

$$
v_{*}\left(T^{*}\right)=\left(\begin{array}{c}
c_{0}+c_{1} \\
c_{0}
\end{array}\right) .
$$

Поэтому

$$
v_{*}(T) \leqslant\left(\begin{array}{c}
c_{0}+c_{1} \\
c_{0}
\end{array}\right) .
$$

\section{3. Метод ветвей и границ для задачи о ранце}

\section{1. Постановка задачи о ранце}

Задача о ранце формально описывается следующим образом:

$$
\begin{gathered}
\sum_{i=1}^{n} p_{i} x_{i} \rightarrow \max ; \\
\sum_{i=1}^{n} w_{i} x_{i} \leq C ; \\
x_{i} \in\{0,1\}, \quad i \in N .
\end{gathered}
$$


Здесь и далее через $N$ обозначается множество $1, \ldots, n$. В данной постановке через $p_{i}$ и $w_{i}$ обозначены стоимость и вес $i$-го предмета, помещаемого в ранец грузоподъемностью $C$. Задача состоит в определении набора предметов максимальной суммарной стоимости, который можно разместить в ранце.

Для удобства описания метода ветвей и границ введем в рассмотрение более общую постановку задачи о ранце, в которой предполагается, что часть переменных принимает фиксированные значения:

$$
\begin{gathered}
\sum_{i=1}^{n} p_{i} x_{i} \rightarrow \max ; \\
\sum_{i=1}^{n} w_{i} x_{i} \leqslant C ; \\
x_{i}=\theta_{i}, \quad i \in I, \quad \theta_{i} \in\{0,1\} ; \\
x_{i} \in\{0,1\}, \quad i \in N \backslash I,
\end{gathered}
$$

где $I$ - множество индексов всех переменных $x_{i}$, принимающих фиксированные значения $\theta_{i}$. Отметим, что задача (1) является частным случаем задачи (2), когда множество $I$ пусто.

Задаче (2) соответствует следующая линейная задача релаксации:

$$
\begin{aligned}
& \sum_{i=1}^{n} p_{i} x_{i} \rightarrow \max ; \\
& \sum_{i=1}^{n} w_{i} x_{i} \leqslant C ; \\
& x_{i}=\theta_{i}, \quad i \in I, \quad \theta_{i} \in\{0,1\} \\
& 0 \leqslant x_{i} \leqslant 1, \quad i \in N \backslash I .
\end{aligned}
$$

Оптимум задачи (3) не меньше оптимума задачи (2). Поэтому, если оптимальное решение задачи (3) достигается на целочисленном наборе значений $x_{1}, \ldots, x_{n}$, то этот набор является также оптимальным решением задачи (2).

Задача (3) представляет собой одномерную задачу линейного программирования и может быть решена методом Данцига [6] следующим образом. Переменные нумеруются в порядке убывания удельной стоимости:

$$
\frac{p_{1}}{w_{1}} \geqslant \ldots \geqslant \frac{p_{n}}{w_{n}}
$$

Затем определяется номер $s$ дробной переменной по следующему правилу:

$$
s=\min \left\{j \in N \backslash I \backslash \sum_{i \in N \backslash I, i \leqslant j} w_{i}+\sum_{i \in I} \theta_{i} w_{i}>C\right\} .
$$

Если такого $s$ не существует, то есть

$$
\sum_{i \in N \backslash I} w_{i}+\sum_{i \in I} \theta_{i} w_{i} \leqslant C,
$$


то решение задачи (3) определяется соотношениями:

$$
x_{i}= \begin{cases}\theta_{i}, & i \in I, \\ 1, & i \in N \backslash I .\end{cases}
$$

В противном случае решение задачи (3) задается следующим образом:

$$
\begin{aligned}
& x_{i}= \begin{cases}\theta_{i}, & i \in I, \\
1, & i<s, i \in N \backslash I, \\
0, & s<i, i \in N \backslash I,\end{cases} \\
& x_{s}=\frac{1}{w_{s}}\left(C-\sum_{i \in N \backslash I, i<s} w_{i}-\sum_{i \in I} \theta_{i} w_{i}\right) .
\end{aligned}
$$

Определение дробной переменной и решение задачи релаксации требует линейного относительно $n$ числа операций.

\section{2. Метод ветвей и границ для решения задачи о ранце}

Дадим общее описание метода ветвей и границ $[2,3,6]$ для решения задачи о ранце. В процессе работы алгоритма поддерживаются следующие данные: рекорд, то есть наибольшее найденное на данный момент времени значение целевой функции $f$, рекордное решение, на котором достигается рекорд, и текущий список подзадач, на которые разбита исходная задача. Подзадачи из списка являются задачами вида (2).

Данные: рекорд $f^{0}$, рекордное решение $x^{0}$, список подзадач.

Шаг 1. В список подзадач помещается исходная задача. Рекорд полагается равным 0.

Шаг 2. Если список подзадач пуст, то алгоритм завершается. В противном случае выбирается подзадача $P$ из списка подзадач. Подзадача $P$ удаляется из списка.

Шаг 3. Проверяется, выполнены ли для выбранной подзадачи $P$ указанные далее условия отсева. Если одно из условий отсева выполнено, то осуществляется переход к шагу 2. При необходимости на этом шаге также обновляются рекорд и соответствующее рекордное решение.

Шаг 4. Выбранная подзадача подвергается декомпозиции. Для этого выбирается переменная $x_{b}$, называемая переменной ветвления. Подзадача $P$ вида (2) разбивается на две подзадачи $P_{0}$ и $P_{1}$, получаемые присваиванием переменной $x_{b}$ значений 0 и 1 соответственно:

подзадача $P_{0}$ :

$$
\begin{aligned}
& \sum_{i=1}^{n} p_{i} x_{i} \rightarrow \max ; \\
& \sum_{i=1}^{n} w_{i} x_{i} \leqslant C ; \\
& x_{i}=\theta_{i}, \quad i \in I, \quad x_{b}=0, \\
& x_{i} \in\{0,1\}, \quad i \in N \backslash\{I \cup b\} .
\end{aligned}
$$


подзадача $P_{1}$ :

$$
\begin{aligned}
& \sum_{i=1}^{n} p_{i} x_{i} \rightarrow \max ; \\
& \sum_{i=1}^{n} w_{i} x_{i} \leqslant C ; \\
& x_{i}=\theta_{i}, \quad i \in I, \quad x_{b}=1, \\
& x_{i} \in\{0,1\}, \quad i \in N \backslash\{I \cup b\} .
\end{aligned}
$$

Построенные подзадачи $P_{0}$ и $P_{1}$ помещаются в список подзадач и осуществляется переход к шагу 2.

Результатом работы алгоритма является окончательное рекордное решение. Заметим, что работа описанного нами алгоритма существенным образом зависит от процедуры выбора очередной подзадачи из списка подзадач и процедуры выбора переменной ветвления для декомпозиции выбранной подзадачи. Алгоритм, для которого данные процедуры строго определены, будем называть вариантом метода ветвей и границ.

Вариант МВГ будем называть стандартным, если условиями отсева выбранной подзадачи $P$ вида (2) являются следующие условия:

R1: $\sum_{i \in I} \theta_{i} w_{i}>C$, то есть подзадача $P$ не имеет решения;

R2: Оптимальное решение соответствующей подзадачи $P$ задачи релаксации (3) является целочисленным, то есть данное решение также является оптимальным решением подзадачи $P$, поэтому подзадача $P$ не нуждается в дальнейшей декомпозиции.

R3: Оптимальное значение целевой функции $f$ в соответствующей подзадаче $P$ задачи релаксации (3) не превосходит значение текущего рекорда, тем самым оптимальное значение целевой функции $f$ для подзадачи $P$ также заведомо не превосходит значение текущего рекорда.

Если выполнено условие R2 и оптимальное значение целевой функции $f$ для соответствующей подзадачи $P$ задачи релаксации превосходит текущий рекорд, то данное значение целевой функции становится рекордом, а рекордным решением становится соответствующее оптимальное решение.

Вариант МВГ будем называть ослабленным, если условиями отсева выбранной подзадачи $P$ вида (2) являются условие R1 и условие

R2': $\sum_{i \in I} \theta_{i} w_{i}+\sum_{i \in N \backslash I} w_{i} \leqslant C$, то есть оптимальным решением подзадачи $P$ является набор значений $x_{1}, \ldots, x_{n}$ такой, что $x_{i}=\theta_{i}$ при $i \in I$ и $x_{i}=1$ при $i \in N \backslash I$.

Для данного варианта МВГ рекорд и рекордное решение обновляются, если выполнено условие R2' и оптимальное значение целевой функции $f$ для подзадачи $P$ превосходит текущий рекорд (рекордом становится данное значение целевой функции, а рекордным решением становится оптимальное решение подзадачи $P$ ).

3 Дискретная математика, т.22 №1 


\section{4. Оценка сложности метода ветвей и границ}

Процесс решения задачи о ранце методом ветвей и границ можно представить в виде дерева ветвления, вершинам которого соответствуют рассматриваемые в процессе решения подзадачи. Вершина, соответствующая подзадаче, соединяется дугами с вершинами, соответствующими подзадачам, полученным из этой подзадачи в результате декомпозиции. Дуга, ведущая в вершину, соответствующую подзадаче, полученной присваиванием переменной ветвления значения 0 (значения 1), помечается символом 0 (символом 1). Корнем дерева ветвления полагается вершина, соответствующая исходной задаче. Отметим, что дерево ветвления решения задачи о ранце методом ветвей и границ является, очевидно, би-деревом. Сложность решения задачи методом ветвей и границ будем определять как число концевых вершин в дереве ветвления. Заметим, что это число равно по порядку общему числу вершин в дереве ветвления, то есть числу рассматриваемых в процессе решения задачи подзадач.

\section{1. Оценки сложности ослабленного варианта метода ветвей и границ}

\subsection{1. Оценки сложности через соотношения величин весов и грузоподъемности ранца}

Пусть веса упорядочены в порядке убывания: $w_{1} \geqslant \ldots \geqslant w_{n}$. Определим величины $s$ и $t$ следующим образом:

$$
\begin{aligned}
& s=\min \left\{k \in N: \sum_{i=1}^{k} w_{i}>C\right\}, \\
& t=\min \left\{k \in N: \sum_{i=n-k+1}^{n} w_{i}>C\right\} .
\end{aligned}
$$

Предложение 4. Пусть T - дерево ветвления для решения задачи (1) ослабленньмм вариантом МВГ. Тогда для любой вершины х из множества $V_{*}^{1}(T)$ справедливо неравенство

$$
s \leqslant e_{1}(x) \leqslant t
$$

и для любой вершины у из множества $V_{*}^{0}(T)$ справедливо неравенство

$$
n+1-t \leqslant e_{0}(y) \leqslant n+1-s,
$$

где величины s и t определяются соотношениями (4), (5).

Доказательство. Пусть $x$ - произвольная вершина из $V_{*}^{1}(T)$, и соответствующая этой вершине подзадача $P$ имеет вид (2). Для множеств индексов фиксированных переменных, принимающих значения 0 и 1 , введем обозначения

$$
\begin{aligned}
& I_{0}=\left\{i \in I \mid \theta_{i}=0\right\}, \\
& I_{1}=\left\{i \in I \mid \theta_{i}=1\right\} .
\end{aligned}
$$

Обозначим через $\widehat{P}$ подзадачу, из которой была получена подзадача $P$ в результате декомпозиции. Так как $x \in V_{*}^{1}(T)$, подзадача $P$ не может удовлетворять условию отсева 
R2', поскольку в противном случае этому же условию удовлетворяет подзадача $\widehat{P}$. Следовательно, подзадача $P$ должна удовлетворять условию отсева R1, то есть

$$
\sum_{i \in I} \theta_{i} w_{i}>C
$$

что равносильно неравенству

$$
\sum_{i \in I_{1}} w_{i}>C
$$

Так как $w_{1} \geqslant \ldots \geqslant w_{n}$, справедливы неравенства

$$
\sum_{i=1}^{\left|I_{1}\right|} w_{i} \geqslant \sum_{i \in I_{1}} \theta_{i} w_{i}>C .
$$

Согласно (4), справедливо равенство

$$
e_{1}(x)=\left|I_{1}\right| \geqslant s .
$$

Пусть $x_{j}-$ переменная ветвления для подзадачи $\hat{P}$. Так как $x \in V_{*}^{1}(T)$, справедливо равенство $\theta_{j}=1$. Обозначим через $J_{1}$ множество $I_{1} \backslash\{j\}$. Подзадача $\widehat{P}$ не удовлетворяет условию отсева R1, поэтому

$$
\sum_{i \in J_{1}} w_{i} \leqslant C
$$

Так как $w_{1} \geqslant \ldots \geqslant w_{n}$, справедливы неравенства

$$
\sum_{i=n-\left|J_{1}\right|+1}^{n} w_{i} \leqslant \sum_{i \in J_{1}} w_{i} \leqslant C .
$$

Из (5) следует, что

$$
e_{1}(x)=\left|I_{1}\right|=\left|J_{1}\right|+1 \leqslant t .
$$

Пусть теперь подзадача $P$ вида (2) соответствует произвольной вершине $y$ из $V_{*}^{0}(T)$. Пусть также подзадача $P$ получена в результате декомпозиции из подзадачи $\hat{P}$. Так как $y \in V_{*}^{0}(T)$, подзадача $P$ не может удовлетворять условию отсева R1, поскольку в противном случае этому же условию удовлетворяет подзадача $\widehat{P}$. Следовательно, подзадача $P$ удовлетворяет условию отсева R2'. Поэтому

$$
\sum_{i \in I} \theta_{i} w_{i}+\sum_{i \in N \backslash I} w_{i} \leqslant C,
$$

что эквивалентно неравенству

$$
\sum_{i \in N \backslash I_{0}} w_{i} \leqslant C .
$$

Так как $w_{1} \geqslant \ldots \geqslant w_{n}$, справедливы неравенства

$$
\sum_{i=n-\left|N \backslash I_{0}\right|+1}^{n} w_{i} \leqslant \sum_{i \in N \backslash I_{0}} w_{i} \leqslant C .
$$


Из (5) следует, что

$$
n-e_{0}(y)=\left|N \backslash I_{0}\right| \leqslant t-1,
$$

поэтому

$$
e_{0}(y) \geqslant n+1-t \text {. }
$$

Так как $y \in V_{*}^{0}(T)$, выполняется равенство $\theta_{j}=0$, где $j-$ индекс переменной ветвления для подзадачи $\widehat{P}$. Обозначим через $J_{0}$ множество $I_{0} \backslash\{j\}$. Для подзадачи $\widehat{P}$ не выполнено условие отсева R2', поэтому

$$
\sum_{i \in N \backslash J_{0}} w_{i}>C
$$

Так как $w_{1} \geqslant \ldots \geqslant w_{n}$, справедливы неравенства

$$
\sum_{i=1}^{\left|N \backslash J_{0}\right|} w_{i} \geqslant \sum_{i \in N \backslash J_{0}} w_{i}>C .
$$

Согласно (4),

$$
n-e_{0}(y)+1=\left|N \backslash J_{0}\right| \geqslant s .
$$

Таким образом,

$$
e_{0}(y) \leqslant n+1-s \text {. }
$$

Тем самым предложение доказано.

Из доказанного предложения и предложений 2, 3 вытекает следующее утверждение.

Предложение 5. Сложность $S$ решения задачи (1) ослабленным вариантом МВГ удовлетворяет неравенствам

$$
\left(\begin{array}{c}
n+1-t+s \\
s
\end{array}\right) \leqslant S \leqslant\left(\begin{array}{c}
n+1-s+t \\
t
\end{array}\right),
$$

где величины s и t определяются соотношениями (4), (5) $и\left(\begin{array}{l}a \\ b\end{array}\right)=1$ при $a<b$.

Несложно заметить, что в соотношениях (6) верхняя оценка для $S$ совпадает с нижней тогда и только тогда, когда $s=t$.

\subsection{2. Оценка сложности через соотношения максимального и минимального весов}

Для минимального и максимального весов будем использовать обозначения

$$
\underline{w}=\min _{i \in N} w_{i}, \quad \bar{w}=\max _{i \in N} w_{i}
$$

Пусть $T$ - дерево ветвления для решения задачи (1) произвольным ослабленным вариантом МВГ, $x$ - концевая вершина дерева $T$, которой соответствует подзадача $P$, имеющая 
вид (2). Обозначим через $\tilde{\sigma}(x)=\left(\sigma_{1}(x), \ldots, \sigma_{n}(x)\right)$ булев набор длины $n$, определяемый следующим образом:

$$
\sigma_{i}(x)= \begin{cases}\theta_{i}, & i \in I, \\ 0, & i \notin I, x \in V_{*}^{1}(T), \quad i=1, \ldots, n . \\ 1, & i \notin I, x \in V_{*}^{0}(T),\end{cases}
$$

Заметим, что для любых различных концевых вершин $x, y$ дерева $T$ наборы $\tilde{\sigma}(x), \tilde{\sigma}(y)$ различаются в компоненте, номер которой равен индексу переменной ветвления для подзадачи, соответствующей корню наименьшего би-поддерева в дереве $T$, содержащего обе вершины $x$ и $y$. Таким образом, если $x \neq y$, то $\tilde{\sigma}(x) \neq \tilde{\sigma}(y)$. Пусть

$$
W(x)=\sum_{i=1}^{n} \sigma_{i}(x) w_{i}
$$

и $\|\tilde{\sigma}(x)\|-$ число единичных компонент набора $\tilde{\sigma}(x)$. Из доказательства предложения 5 следует, что $W(x)>C$ для любой вершины $x$ из $V_{*}^{1}(T)$ и $W(y) \leqslant C$ для любой вершины $y$ из $V_{*}^{0}(T)$. Таким образом, для любых $x \in V_{*}^{1}(T), y \in V_{*}^{0}(T)$ выполняется неравенство $W(y)<W(x)$, тем самым либо $\tilde{\sigma}(y)<\tilde{\sigma}(x)$, либо наборы $\tilde{\sigma}(x), \tilde{\sigma}(y)$ несравнимы.

Предложение 6. Пусть $T$ - дерево ветвления для решения задачи (1) ослабленным вариантом МВГ. Тогда

(а) для любых вершин $x, y$ из $V_{*}^{1}(T)$ таких, что $\tilde{\sigma}(x) \leqslant \tilde{\sigma}(y)$, выполняется неравенство

$$
\|\tilde{\sigma}(y)\|-\|\tilde{\sigma}(x)\|<\bar{w} / \underline{w}
$$

(б) для любых вершин $x, y$ из $V_{*}^{0}(T)$ таких, что $\tilde{\sigma}(x) \leqslant \tilde{\sigma}(y)$, выполняется неравенство

$$
\|\tilde{\sigma}(y)\|-\|\tilde{\sigma}(x)\|<\bar{w} / \underline{w} .
$$

Доказательство. Пусть $x \in V_{*}^{1}(T)$, соответствующая вершине $x$ подзадача $P$ имеет вид (2), $\widehat{P}$ - подзадача, из которой получена в результате декомпозиции подзадача $P$, и $x_{j}-$ переменная ветвления для подзадачи $\widehat{P}$. Подзадача $\widehat{P}$ не удовлетворяет условию отсева R1, поэтому

$$
\sum_{i \in I \backslash\{j\}} \theta_{i} w_{i} \leqslant C
$$

Следовательно,

$$
W(x)=\sum_{i \in I} \theta_{i} w_{i} \leqslant C+w_{j} \leqslant C+\bar{w} .
$$

Кроме того, как отмечено выше, $W(x)>C$. Таким образом,

$$
C<W(x) \leqslant C+\bar{w}
$$

для любой вершины $x \in V_{*}^{1}(T)$. Поэтому для любых $x, y \in V_{*}^{1}(T)$ выполняется неравенство

$$
|W(x)-W(y)|<\bar{w}
$$


С другой стороны, если $\tilde{\sigma}(x) \leqslant \tilde{\sigma}(y)$, то, очевидно,

$$
W(y)-W(x) \geqslant(\|\tilde{\sigma}(y)\|-\|\tilde{\sigma}(x)\|) \underline{w} .
$$

Таким образом, в этом случае $(\|\tilde{\sigma}(y)\|-\|\tilde{\sigma}(x)\|) \underline{w}<\bar{w}$, то есть

$$
\|\tilde{\sigma}(y)\|-\|\tilde{\sigma}(x)\|<\bar{w} / \underline{w} .
$$

Пусть теперь $x \in V_{*}^{0}(T)$. Подзадача $\hat{P}$ не удовлетворяет условию отсева R2', поэтому

$$
w_{j}+\sum_{i \in I \backslash\{j\}} \theta_{i} w_{i}+\sum_{i \in N \backslash I} w_{i}>C .
$$

Следовательно,

$$
W(x)=\sum_{i \in I \backslash\{j\}} \theta_{i} w_{i}+\sum_{i \in N \backslash I} w_{i}>C-w_{j} \geqslant C-\bar{w} .
$$

Кроме того, как отмечено выше, $W(x) \leqslant C$. Таким образом, для любой вершины $x \in V_{*}^{0}(T)$ справедливо неравенство

$$
C-\bar{w}<W(x) \leqslant C .
$$

Следовательно,

$$
|W(x)-W(y)|<\bar{w}
$$

для любых $x, y \in V_{*}^{0}(T)$.

Поэтому, аналогично случаю вершин из $V_{*}^{1}(T)$, из неравенства $\tilde{\sigma}(x) \leqslant \tilde{\sigma}(y)$ следует, что

$$
\|\tilde{\sigma}(y)\|-\|\tilde{\sigma}(x)\|<\bar{w} / \underline{w} .
$$

Из предложения 6 вытекает, что как любая цепочка попарно сравнимых наборов $\tilde{\sigma}(x)$ таких, что $x \in V_{*}^{1}(T)$, так и любая цепочка попарно сравнимых наборов $\tilde{\sigma}(x)$ таких, что $x \in V_{*}^{0}(T)$, содержит не более $\lceil\bar{w} / \underline{w}\rceil$ наборов. Пользуясь этим фактом, нетрудно доказать следующее утверждение.

Предложение 7. Пусть T - дерево ветвления для решения задачи (1) ослабленным вариантом МВГ. Тогда множество всех наборов $\tilde{\sigma}(x)$ таких, что $x \in V_{*}^{1}(T)$ или $x \in V_{*}^{0}(T)$, можно покрыть не более чем $\lceil\bar{w} / \underline{w}\rceil$ непересекающимися антицепями.

Из предложения 7 получаем, что множество всех наборов $\tilde{\sigma}(x)$ таких, что $x \in V_{*}(T)$, можно покрыть не более чем $2\lceil\bar{w} / \underline{w}\rceil$ непересекающимися антицепями. С другой стороны, нетрудно убедиться, что максимальная суммарная мощность произвольного числа непересекающихся антицепей в $n$-мерном единичном кубе равна максимальной суммарной мощности такого же числа различных слоев этого куба. Таким образом, получаем следующее утверждение.

Предложение 8. Сложность решения задачи (1) ослабленным вариантом МВГ не превосходит

$$
\sum_{i=\lfloor n / 2\rfloor-\lceil\bar{w} / \underline{w}\rceil+1}^{\lfloor n / 2\rfloor+\lceil\bar{w} / \underline{w}\rceil}\left(\begin{array}{l}
n \\
i
\end{array}\right) .
$$




\section{2. Верхние оценки сложности для задачи о ранце}

Отметим, что любому стандартному варианту $\mathscr{A}$ метода ветвей и границ можно сопоставить ослабленный вариант $\mathscr{A}^{*}$, получающийся из $\mathscr{A}$ заменой условия отсева R2 на R2 и удалением условия отсева R3. Очевидно, что сложность решения задачи методом $\mathcal{A}$ не превосходит сложности решения задачи методом $\mathscr{A}^{*}$. Поэтому из предложений 5 и 8 вытекает следующее утверждение.

Теорема 1. Сложность $S$ решения задачи (1) стандартным вариантом МВГ удовлетворяет неравенствам

$$
\begin{aligned}
& S \leqslant\left(\begin{array}{c}
n+1-s+t \\
t
\end{array}\right), \\
& S \leqslant \sum_{i=\lfloor n / 2\rfloor-\lceil\bar{w} / \underline{w}\rceil+1}^{\lfloor n / 2\rfloor+\lceil\bar{w} / \underline{w}\rceil}\left(\begin{array}{l}
n \\
i
\end{array}\right),
\end{aligned}
$$

где величины s и $t$ определяются соотношениями (4) $и$ (5).

Из оценки (8) вытекает, что сложность решения задачи о ранце стандартным вариантом МВГ не превосходит по порядку $2^{n} \bar{w} /(\underline{w} \sqrt{n})$.

Из оценки (7) следует, что если величина $t$ ограничена, то сложность решения задачи о ранце стандартным вариантом МВГ ограничена сверху полиномом от числа переменных задачи. Ограниченность $t$ может иметь место, например, если отношение $C / \underline{w}$ ограничено сверху константой при росте $n$, так как $t \leqslant C / \underline{w}$.

Следствие 1. Если величина задачи (1) стандартным вариантом МВГ ограничена сверху полиномом

$$
P(n)=\frac{1}{t_{0} !} \prod_{i=1}^{t_{0}}(n+1-s+i)
$$

степени $t_{0}$, зависящим от размерности п данной задачи.

\section{3. Верхняя и нижняя оценки сложности метода ветвей и границ для задачи о сумме подмножеств}

Для задачи о ранце можно рассмотреть важный частный случай, когда стоимость каждого предмета равна его весу. Этот частный случай называется задачей о сумме подмножеств $[2,3]$. Математически задача о сумме подмножеств задается следующим образом:

$$
\begin{aligned}
\sum_{i=1}^{n} w_{i} x_{i} & \rightarrow \max ; \\
\sum_{i=1}^{n} w_{i} x_{i} & \leqslant C ; \\
x_{i} & \in\{0,1\} .
\end{aligned}
$$

Задачу о сумме подмножеств будем называть невырожденной, если ни на одном наборе значений переменных $x_{1}, \ldots, x_{n}$ сумма $\sum_{i=1}^{n} w_{i} x_{i}$ не равна $C$. 
Предложение 9. Для любого стандартного варианта МВГ А сложность решения невырожденной задачи о сумме подмножеств методом А совпадает со сложностью решения этой задачи методом $\AA^{*}$.

Доказательство. Пусть $P$ - произвольная невырожденная задача о сумме подмножеств. Для доказательства предложения достаточно показать эквивалентность наборов условий $\mathrm{R} 1, \mathrm{R} 2{ }^{\prime}$ и R1, R2, R3 для любой подзадачи $P^{\prime}$ задачи $P$. Так как условие R2' является частным случаем условия R2, из выполнения для $P^{\prime}$ какого-то из условий R1, R2' следует выполнение одного из условий R1, R2, R3. Покажем теперь, что выполнение для $P^{\prime}$ одного из условий R1, R2, R3 влечет выполнение одного из условий R1, R2'. Для этого достаточно показать, что если $P^{\prime}$ не удовлетворяет ни одному из условий $\mathrm{R} 1$, R2 $2^{\prime}$, то $P^{\prime}$ не удовлетворяет также ни одному из условий R1, R2, R3. Пусть подзадача $P^{\prime}$ имеет вид

$$
\begin{gathered}
\sum_{i=1}^{n} w_{i} x_{i} \rightarrow \max ; \\
\sum_{i=1}^{n} w_{i} x_{i} \leqslant C ; \\
x_{i}=\theta_{i}, \quad i \in I, \\
x_{i} \in\{0,1\}, \quad i \in N \backslash I
\end{gathered}
$$

и не удовлетворяет ни одному из условий отсева R1, R2'. Задаче (10) соответствует линейная задача релаксации

$$
\begin{gathered}
\sum_{i=1}^{n} w_{i} x_{i} \rightarrow \max ; \\
\sum_{i=1}^{n} w_{i} x_{i} \leqslant C ; \\
x_{i}=\theta_{i}, \quad i \in I, \\
0 \leqslant x_{i} \leqslant 1, \quad i \in N \backslash I .
\end{gathered}
$$

Поскольку $P^{\prime}$ не удовлетворяет ни одному из условий R1, R2', справедливы неравенства

$$
\sum_{i \in I} \theta_{i} w_{i} \leqslant C<\sum_{i \in I} \theta_{i} w_{i}+\sum_{i \in N \backslash I} w_{i} .
$$

Следовательно, оптимальное значение целевой функции $f$ для задачи (11) равно $C$. С другой стороны, в силу невырожденности задачи $P$ текущее значение рекорда не может быть равным $C$. Таким образом, для $P^{\prime}$ не может быть выполнено условие R3. Кроме того, из невырожденности задачи $P$ вытекает невырожденность задачи $P^{\prime}$. Поэтому условие R2 может выполняться для $P^{\prime}$ лишь в случае, когда $P^{\prime}$ удовлетворяет условию R2'. Тем самым предложение доказано.

Из предложений 5 и 9 непосредственно вытекает следующая теорема.

Теорема 2. Для сложности $S$ решения невырожденной задачи (9) о сумме подмножеств стандартным вариантом МВГ справедливы неравенства

$$
\left(\begin{array}{c}
n+1-t+s \\
s
\end{array}\right) \leqslant S \leqslant\left(\begin{array}{c}
n+1-s+t \\
t
\end{array}\right),
$$


где величины s и t определяются соотношениями (4), (5).

Тривиальным следствием теоремы 2 является известный факт, установленный в [8]: число вершин в дереве ветвления для решения задачи

$$
\begin{aligned}
& \sum_{i=1}^{n} 2 x_{i} \rightarrow \max , \\
& \sum_{i=1}^{n} 2 x_{i} \leqslant 2\lfloor n / 2\rfloor+1
\end{aligned}
$$

произвольным стандартным вариантом МВГ равно

$$
2\left(\begin{array}{c}
n+1 \\
\lfloor n / 2\rfloor+1
\end{array}\right)-1 .
$$

\section{5. Заключение}

В данной работе получены оценки сложности решения задачи о ранце методом ветвей и границ. Из этих оценок вытекает, что в случае, если отношение между весами предметов ограничено некоторой константой, то для широкой совокупности вариантов МВГ сложность решения задачи о ранце не превосходит по порядку $2^{n} / \sqrt{n}$. С другой стороны, известно, что в произвольном случае существуют варианты МВГ, для которых максимальная сложность решения задачи о ранце равна по порядку $2^{n}$. Поэтому представляется интересным описание всех вариантов МВГ, для которых сложность решения произвольной задачи о ранце не превосходит по порядку $2^{n} / \sqrt{n}$. Пример такого варианта МВГ найден в [9].

\section{Список литературы}

1. Яблонский С. В., Введение в дискретную математику. Наука, Москва, 1986.

2. Kellerer H., Pferschy U., Pisinger D., Knapsack problems. Springer, Berlin, 2004.

3. Martello S., Toth P., Knapsack problems. Wiley, New York, 1990.

4. Kolesar P. J., A branch and bound algorithm for the knapsack problem. Management Sci. (1967) 13, 723-735.

5. Greenberg H., Hegerich R. L., A branch and bound algorithm for the knapsack problem. Management Sci. (1970) 16, 327-332.

6. Сигал И. Х., Иванова А. П., Введение в прикладное дискретное программирование. Физматлит, Москва, 2002.

7. Гришухин В. П., Эффективность метода ветвей и границ в задачах с булевыми переменными. В сб.: Исследования по дискретной оптимизации. Наука, Москва, 1976, с. 203-230.

8. Финкельштейн Ю. Ю., Приближенные методы и прикладные задачи дискретного программирования. Наука, Москва, 1976.

9. Колпаков Р. М., Посыпкин М. А., Сигал И. Х., О сложности решения задачи о булевом ранце. В сб.: Дискретные модели в теории управляющих систем. МАКС Пресс, Москва, 2006, с. 166171.

Статья поступила 1.04.2009. 